\title{
The Application of Computed Tomography Angiography in Aortic Dissection
}

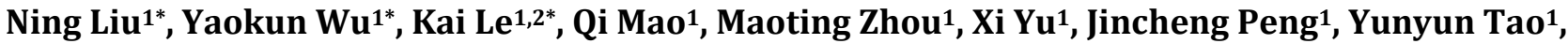 \\ Xueqin Gong1, Yongjun Ren1, Hao Xu1 ${ }^{1}$ Xuli Min1, Jing Zheng1, Weicheng Wang1, Linjun Ma1, \\ Lin Yang1\#
}

${ }^{1}$ Medical Imaging Key Laboratory of Sichuan Province; Medical Research Center; Department of Radiology, The Affiliated Hospital of North Sichuan Medical College, Nanchong, China

${ }^{2}$ Department of Radiology, Xichong County People's Hospital, Xichong, China

Email: \#linyangmd@163.com

How to cite this paper: Liu, N., Wu, Y.K., Le, K., Mao, Q., Zhou, M.T., Yu, X., Peng, J.C., Tao, Y.Y., Gong, X.Q., Ren, Y.J., Xu, H., Min, X.L., Zheng, J., Wang, W.C., Ma, L.J. and Yang, L. (2021) The Application of Computed Tomography Angiography in Aortic Dissection. World Journal of Cardiovascular Diseases, 11, 464-475. https://doi.org/10.4236/wjcd.2021.119044

Received: August 30, 2021

Accepted: September 27, 2021

Published: September 30, 2021

Copyright $\odot 2021$ by author(s) and Scientific Research Publishing Inc. This work is licensed under the Creative Commons Attribution International License (CC BY 4.0).

http://creativecommons.org/licenses/by/4.0/

\begin{abstract}
Aortic dissection $(\mathrm{AD})$ is a life-threatening clinical emergency requiring rapid diagnosis and effective intervention to improve patient survival and prognosis. Computed tomography angiography (CTA) can be used to diagnose AD accurately and quickly, making it the first choice for diagnosing $\mathrm{AD}$ in an emergency. This article reviews the application of CTA in the diagnosis and treatment of AD.
\end{abstract}

\section{Keywords}

Computed Tomography Angiography, Aortic Dissection, Diagnosis, Follow-Up

\section{Introduction}

Aortic dissection (AD) is a life-threatening cardiovascular critical illness mainly caused by the formation of true and false lumens due to the separation of the intima and media after the blood enters the media upon tearing of the aortic intima [1] [2] [3]. A few patients may also experience secondary dissection due to aortic intramural hematoma [4] [5] [6]. With the continuous inflow of blood, the pressure in the false lumen gradually rises, and the false lumen extends rapidly along the longitudinal axis of the aorta, which may eventually result in rupture of the aortic wall [3] [7]. The risk factors for AD include hypertension, aneurysm of the aorta and bicuspid aortic valves, smoking, atherosclerosis, *These authors contributed equally.

\#Corresponding author. 
trauma, and genetic connective tissue disorders such as Marfan, Loeys-Dietz or Ehlers-Danlos syndrome [2] [8] [9]. The incidence of AD in the general population is approximately $6 / 100,000$ per year and tends to increase with age [2] [10] [11]. The overall mortality of acute $\mathrm{AD}$ in the first International Registry of Acute Aortic Dissection (IRAD) publication was $27.7 \%$; the early mortality of acute $\mathrm{AD}$ patients without timely treatment was $1 \%-2 \%$ per hour [3] [8]. Timely and accurate diagnosis is helpful in reducing the mortality and improving the prognosis of $\mathrm{AD}$ patients. Computed tomography angiography (CTA) is the first choice for diagnosing $\mathrm{AD}$ in an emergency. This article mainly reviews the application of CTA in the diagnosis and treatment of $\mathrm{AD}$.

\section{AD Classification}

There are many classification schemes for AD. The Stanford classification and the DeBakey classification, both of which involve the location of the intimal rupture and the scope of aortic involvement, are widely used in the clinic [1] [2] [5] [7] [12] [13] [14]. A Stanford type A dissection involves the ascending aorta, regardless of the location of the rupture; a Stanford type B dissection only involves the descending aorta distal to the opening of the left subclavian artery [2] [5] [14]. A tear in a DeBakey type I dissection is located in the ascending aorta and involves the descending aorta as well; a DeBakey type II dissection involves only the ascending aorta; and a rupture in a type III dissection is located at the distal opening of the left subclavian artery, involving the descending aorta and abdominal aorta downward. A dissection limited to the descending thoracic aorta is classified as type IIIa, and one also involving the abdominal aorta is classified as type IIIb [5] [14].

Some scholars have also proposed other classifications [14] [15] [16] [17], such as the " $3 \mathrm{~N} 3 \mathrm{~V}$ " classification for endovascular graft exclusion. Based on the $3 \mathrm{~N} 3 \mathrm{~V}$ classification, the dynamic pathophysiological $\mathrm{NxNyB}$ classification, determined according to the difference in blood flow velocity between different regions of the aorta, has also been proposed [16]. Recently, experts proposed the "301 classification" [17] by modifying the Stanford type B to facilitate endovascular repair regimen development, prognostic evaluation, and follow-up for type B AD patients.

\section{CTA Examination Technology}

CTA is the most widely used examination for the diagnosis and treatment of $\mathrm{AD}$, especially for patients planning to undergo endovascular treatment [2] [5] [14] [18] [19] [20] [21] [22] [23]. CT scanning parameters can be set comprehensively according to patient height, body mass, heart rate, prospective electrocardiographic (ECG) gating, and retrospective ECG gating.

\subsection{Thin Layer Acquisition}

Multislice CTA is characterized by a fast scanning speed, thin layer acquisition 
and postprocessing reconstruction [21] [22]. The endovascular repair guideline for descending thoracic aortic aneurysms published by the Society for Vascular Surgery (SVS) has recommended thin-layer CTA of the whole aorta for patients undergoing thoracic endovascular aortic repair (TEVAR). Otherwise, tiny aortic ruptures may be missed, and the exact relationships between ruptures and branch arteries can be difficult to determine [19] [21].

\subsection{Multiphasic CTA Protocol}

A multiphasic CTA protocol is valuable in the diagnosis and follow-up of $\mathrm{AD}$ [22] [24] [25]. For postoperative follow-up, arterial and delayed phase scanning are usually recommended because endoleaks have variable flow rates [22] [25]. Delayed phase scanning is more beneficial for displaying internal leakage with slow blood flow. The scanning delay often used for the delayed phase ranges from 60 to 120 seconds after contrast agent injection [25]. Some institutions employ a late delayed phase of 300 seconds to better visualize low-flow endoleaks [25].

In addition, the absence of contrast enhancement on arterial and early delayed phase CTA does not indicate complete thrombosis in the false lumen given the possibility of a low-flow state. In such cases, late delayed phase CTA demonstrates higher sensitivity in the detection of partial thrombosis [24]. The scanning range is usually from the upper thoracic orifice to the pelvic floor.

\subsection{Contrast Agent}

Contrast agents should be used in reference to the relevant guidelines in combination with the patient's weight and renal function. Double-syringe high-pressure injectors are recommended. High-concentration nonionic iodinated contrast agent $(60-140 \mathrm{~mL})$ is injected at a rate of $4-6 \mathrm{~mL} / \mathrm{s}$ into the cubital fossa with an intravenous trocar (18-20 G) [21] [22] [26]. Compared with a fixed scanning delay, scanning with contrast agent concentration tracking makes it easier to accurately determine the peak contrast agent concentration in the aorta and achieve better image quality. Therefore, scanning is usually triggered by the tracked contrast agent concentration after injection of contrast agent [22] [26].

\subsection{ECG Gating}

When non-ECG-triggered scanning is used, motion artifacts tend to occur at the root of the ascending aorta due to the influence of the aortic wall and cardiac impulse, thus leading to false positives. Therefore, ECG-triggered scanning is often used, especially for Stanford type A AD patients [2] [21] [26] [27] [28]. Schernthaner RE et al. reported that compared with nongated CTA at the same radiation dose, ECG-gated CTA of the entire aorta reduces motion artifacts and results in a higher diagnostic confidence with the same signal-to-noise ratios [29].

The radiation dose of CTA with prospective ECG gating is lower than that of 
CTA with retrospective ECG gating [26] [29] [30]. Retrospective gating depicts the complete cardiac cycle. In CTA with retrospective ECG gating, CTA images can be dynamically reconstructed at any time phase, dynamic information about aortic wall motion during the cardiac cycle can be observed, and the aortic valve can be evaluated [29].

\subsection{Dual-Source CT (DSCT)}

DSCT adopts dual X-ray sources and dual detection systems, resulting in a qualitative leap in scanning speed. Second- and third-generation DSCT has less strict requirements on heart rate, and diagnostic images can be obtained from most patients without using $\beta$-blockers, making DSCT especially suitable for cardiac and coronary artery examinations [31] [32]. DSCT greatly increases the coverage area in the form of a fan-shaped X-ray distribution in a single plane, which reduces the radiation dose received by patients and the motion artifacts of the aortic root caused by cardiac impulses [33] [34] [35] [36] [37]. DSCT combined with ECG gating can collect data at different time phases of the cycle and reconstruct CTA images of different periods [38] [39] [40]. Continuous multiphase reconstruction can display the changes in the aortic morphology and blood flow in a cinematic sequence and improve the diagnosis of $\mathrm{AD}$ to the level of dynamic evaluation [41].

\section{AD Diagnosis}

The characteristic features of $\mathrm{AD}$ on $\mathrm{CT}$ images are an intimal flap shadow and a double-lumen structure in the aortic lumen [1] [30] [42]. Calcification of the aortic wall can often be seen on nonenhanced CT scans. The direct evidence leading to the diagnosis of $\mathrm{AD}$ is the displacement of calcified intima into the vascular lumen, and displacement $>5 \mathrm{~mm}$ is of diagnostic significance. Contrast-enhanced axial CT images can clearly display smoothly curved or S-shaped hypoattenuating intimal flaps. If discontinuous hypoattenuating intimae are found in the lumen on a certain level or the contrast agent connects the two lumens, the position of the $\mathrm{AD}$ rupture can be judged. The following imaging features are helpful to distinguish false from true lumens: 1) There are hemodynamic differences between true and false lumens. The blood velocity in the true lumen is relatively high in the early stage and low in the late stage, while that for the false lumen shows the opposite trend [1] [26] [30]. 2) The density in the true lumen is similar to that of the adjacent normal aorta or left ventricle, but the density in the false lumen is inconsistent. 3) The true lumen is the direct continuation of the adjacent normal aorta or left ventricle, but the false lumen is not [26]. 4) The true lumen in typical $\mathrm{AD}$ is small, showing a horseshoe or oval shape; the false lumen is large and half-moon- or crescent-shaped [26] [30]. 5) The false lumen shows a cobweb sign and a beak sign [1] [26] [30] [43] [44]. The reconstructed images after postprocessing can serve as an important supplement to CT images [30] [41] [45] [46] [47] [48] (Figure 1). 

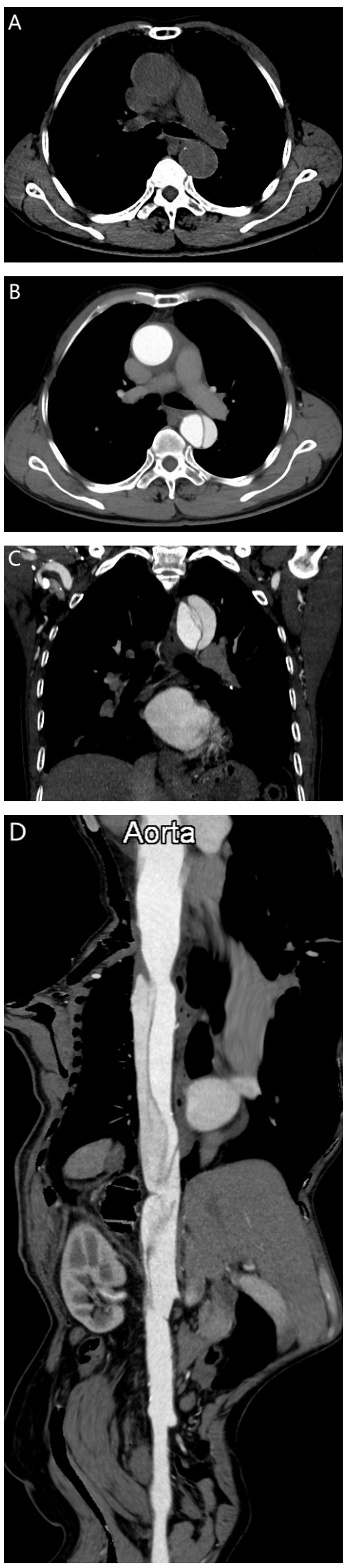


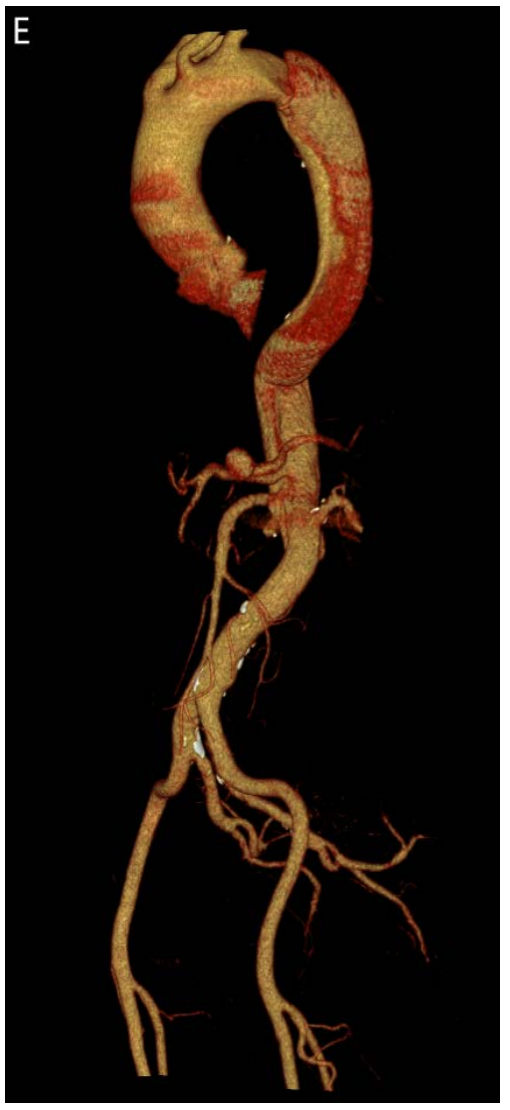

Figure 1. (A) A 69-year-old male, Stanford type B aortic dissection. Nonenhanced axial CT image shows that the calcified intima of the aorta has shifted into the vascular lumen. (B) CTA axial image shows a double-lumen change in the descending aorta and a hypoattenuating intimal flap shadow between the true and false lumens. (C) MPR shows the position of intimal rupture. (D) CPR shows the shape of the intima and the true and false lumens of the aorta. (E) VR shows a double lumen-like change in the lumen from the aortic arch to the abdominal aorta and the rupture at the distal opening of the left subclavian artery. The left renal artery originates from the true lumen, the right renal artery originates from the false lumen, the abdominal trunk originates from the true and false lumens, and the superior mesenteric artery originates from the false lumen.

CTA can be used to diagnose AD quickly and accurately with a sensitivity and specificity close to $100 \%$ and $98 \%$, respectively [49]. Although CTA is a recognized examination standard for $\mathrm{AD}$, it is not absolutely safe. A few complicated ADs may not be found by CTA under special circumstances, so it is necessary to also perform other examinations to confirm or exclude an AD diagnosis [50] [51]. Paulraj [50] reported an 18-year-old male with traumatic type A AD that was not displayed by CTA but was first indicated by transthoracic echocardiogram (TTE) and finally diagnosed by transesophageal echocardiogram (TEE). The results of this study indicated that when the clinical suspicion for $\mathrm{AD}$ is high 
and in the presence of complications of type $\mathrm{A} \mathrm{AD}$, such as aortic regurgitation, it would be prudent to obtain further images through TTE/TEE to rule in or rule out the diagnosis.

Stanford type B AD is mostly treated with TEVAR, which especially depends on imaging indications. In this regard, the following is recommended: 1) rotating to capture images around the rupture to display the maximum diameter of the rupture, the shape and course of the intima, and the true and false lumens; 2) measuring the width of the rupture, the distance between the rupture and the left subclavian artery, and the inner diameter of the aorta; and 3) clearly displaying the relationships between the rupture and the three main vessels on the aortic arch (i.e., originating from the true lumen or the false lumen and a thrombosis inside an endovascularly repaired false lumen) to provide a reference for clinicians to plan TEVAR.

\section{Follow-Up for TEVAR}

Treatments for AD patients include TEVAR, surgery, and hybrid surgery [2] [5] [14]. AD patients need regular follow-up after TEVAR because endoleaks may develop at any time [2] [5] [14] [23] [49] [52]. CTA is the preferred method of image monitoring given its sensitivity in the detection of endoleaks, changes in aortic diameter, evaluation of false lumen thrombosis, and assessment of device migration and integrity. The follow-up plans after TEVAR are not uniform between countries [2] [5] [14] [21] [49] [53] [54]. The imaging follow-up times recommended by the 2014 European Society of Cardiology (ESC) guidelines are as follows: 1 month, 6 months, and 12 months and once per year afterwards. If patients show a stable course without evidence of endoleaks over 2 years, it may be safe to extend the imaging intervals to every 2 years [2].

American guidelines recommend imaging follow-ups at 1 month, 3 or 6 months, and once per year afterwards; in addition, if patients have stable findings $>5$ years, the imaging follow-up may be extended to 18- to 24-month intervals [14] [21] [54].

The following features are the focuses of CT follow-up after TEVAR: blood supply changes in important branches of the aorta; aortic plasticity and false lumen thrombosis; stent displacement, fracture, and deformation; internal leakage (amount and type of internal leakage); new dissection; and postoperative complications [2] [14] [21] [23] [24] [54] [55] [56] [57]. Our previous study investigated the value of CTA in aortic remodeling after TEVAR treatment in 38 Stanford type B AD patients by retrospectively analyzing their clinical data. After TEVAR treatment, the diameters of the true lumens increased, while those of the false lumens decreased obviously in the acute and subacute groups. The maximum diameters of the aorta decreased obviously in the acute group, but they did not change significantly in the subacute group. These results indicated that acute $A D$ may be associated with better aortic remodeling than subacute AD after TEVAR [55]. 


\section{Conclusion}

$\mathrm{AD}$ is a critical, life-threatening cardiovascular disease, requiring timely and effective clinical intervention to improve the chance of survival and prognosis. CTA is the best imaging method for the rapid diagnosis of $\mathrm{AD}$, showing high specificity and sensitivity [14] [21] [58] [59]. The further standardization of scanning technology and the rational application of postprocessing methods are of great value in the diagnosis and follow-up of $\mathrm{AD}$.

\section{Conflicts of Interest}

The authors declare no conflicts of interest regarding the publication of this paper.

\section{References}

[1] McMahon, M.A. and Squirrell, C.A. (2010) Multidetector CT of Aortic Dissection: A Pictorial Review. Radiographics, 30, 445-460.

https://doi.org/10.1148/rg.302095104

[2] Erbel, R., Aboyans, V., Boileau, C., Bossone, E., Bartolomeo, R.D., Eggebrecht, H., et al. (2014) ESC Guidelines on the Diagnosis and Treatment of Aortic Diseases: Document Covering Acute and Chronic Aortic Diseases of the Thoracic and Abdominal Aorta of the Adult. The Task Force for the Diagnosis and Treatment of Aortic Diseases of the European Society of Cardiology (ESC). European Heart Journal, 35, 2873-2926. https://doi.org/10.1093/eurheartj/ehu281

[3] Gawinecka, J., Schönrath, F. and von Eckardstein, A. (2017) Acute Aortic Dissection: Pathogenesis, Risk Factors and Diagnosis. Swiss Medical Weekly, 147, w14489. https://doi.org/10.4414/smw.2017.14489

[4] Clough, R.E. and Nienaber, C.A. (2015) Management of Acute Aortic Syndrome. Nature Reviews Cardiology, 12, 103-114. https://doi.org/10.1038/nrcardio.2014.203

[5] JCS Joint Working Group (2013) Guidelines for Diagnosis and Treatment of Aortic Aneurysm and Aortic Dissection (JCS 2011): Digest Version. Circulation Journal, 77, 789-828. https://doi.org/10.1253/circj.CJ-66-0057

[6] Mussa, F.F., Horton, J.D., Moridzadeh, R., Nicholson, J., Trimarchi, S. and Eagle, K.A. (2016) Acute Aortic Dissection and Intramural Hematoma: A Systematic Review. JAMA, 316, 754-763. https://doi.org/10.1001/jama.2016.10026

[7] Criado, F.J. (2011) Aortic Dissection: A 250-Year Perspective. Texas Heart Institute Journal, 38, 694-700.

[8] Evangelista, A., Isselbacher, E.M., Bossone, E., Gleason, T.G., Eusanio, M.D., Sechtem, U., et al. (2018) Insights from the International Registry of Acute Aortic Dissection: A 20-Year Experience of Collaborative Clinical Research. Circulation, 137, 1846-1860. https://doi.org/10.1161/CIRCULATIONAHA.117.031264

[9] Richens, D., Field, M., Neale, M. and Oakley, C. (2002) The Mechanism of Injury in Blunt Traumatic Rupture of the Aorta. European Journal of Cardio-Thoracic Surgery, 21, 288-293. https://doi.org/10.1016/S1010-7940(01)01095-8

[10] Howard, D.P., Banerjee, A., Fairhead, J.F., Perkins, J., Silver, L.E., Rothwell, P.M., et al. (2013) Population-Based Study of Incidence and Outcome of Acute Aortic Dissection and Premorbid Risk Factor Control: 10-Year Results from the Oxford Vascular Study. Circulation, 127, 2031-2037.

https://doi.org/10.1161/CIRCULATIONAHA.112.000483 
[11] Mokashi, S.A. and Svensson, L.G. (2019) Guidelines for the Management of Thoracic Aortic Disease in 2017. General Thoracic and Cardiovascular Surgery, 67, 59-65. https://doi.org/10.1007/s11748-017-0831-8

[12] Murphy, M.C., Castner, C.F. and Kouchoukos, N.T. (2017) Acute Aortic Syndromes: Diagnosis and Treatment. Missouri Medicine, 114, 458-463.

[13] Nienaber, C.A. and Clough, R.E. (2015) Management of Acute Aortic Dissection. The Lancet, 385, 800-811. https://doi.org/10.1016/S0140-6736(14)61005-9

[14] Lombardi, J.V., Hughes, G.C., Appoo, J.J., Bavaria, J.E., Beck, A.W., Cambria, R.P., et al. (2020) Society for Vascular Surgery (SVS) and Society of Thoracic Surgeons (STS) Reporting Standards for Type B Aortic Dissections. The Annals of Thoracic Surgery, 109, 959-981. https://doi.org/10.1016/j.athoracsur.2019.10.005

[15] Sampson, U.K., Norman, P.E., Fowkes, F.G., Aboyans, V., Song, Y.N., Harrell, F.E., et al. (2014) Global and Regional Burden of Aortic Dissection and Aneurysms: Mortality Trends in 21 World Regions, 1990 to 2010. Global Heart, 9, 171-180.e10. https://doi.org/10.1016/j.gheart.2013.12.010

[16] Markl, M., Draney, M.T., Hope, M.D., Levin, J.M., Chan, F.P., Alley, M.T., et al. (2004) Time-Resolved 3-Dimensional Velocity Mapping in the Thoracic Aorta: Visualization of 3-Directional Blood Flow Patterns in Healthy Volunteers and $\mathrm{Pa}$ tients. Journal of Computer Assisted Tomography, 28, 459-468. https://doi.org/10.1097/00004728-200407000-00005

[17] Ge, Y.Y., Rong, D., Ge, X.H., Miao, J.H., Fan, W.D., Liu, X.P., et al. (2020) The 301 Classification: A Proposed Modification to the Stanford Type B Aortic Dissection Classification for Thoracic Endovascular Aortic Repair Prognostication. Mayo Clinic Proceedings, 95, 1329-1341. https://doi.org/10.1016/j.mayocp.2020.03.031

[18] Pape, L.A., Awais, M., Woznicki, E.M., Suzuki, T., Trimarchi, S., Evangelista, A., et al. (2015) Presentation, Diagnosis, and Outcomes of Acute Aortic Dissection: 17-Year Trends From the International Registry of Acute Aortic Dissection. Journal of the American College of Cardiology, 66, 350-358. https://doi.org/10.1016/j.jacc.2015.05.029

[19] Salmasi, M.Y., Al-Saadi, N., Hartley, P., Jarral, O.A., Raja, S., Hussein, M., et al. (2020) The Risk of Misdiagnosis in Acute Thoracic Aortic Dissection: A Review of Current Guidelines. Heart, 106, 885-891. https://doi.org/10.1136/heartjnl-2019-316322

[20] Bossone, E., LaBounty, T.M. and Eagle, K.A. (2018) Acute Aortic Syndromes: Diagnosis and Management, an Update. European Heart Journal, 39, 739-749d. https://doi.org/10.1093/eurheartj/ehx319

[21] Upchurch, G.R., Escobar, G.A., Azizzadeh, A., Beck, A.W., Conrad, M.F., Matsumura, J.S., et al. (2021) Society for Vascular Surgery Clinical Practice Guidelines of Thoracic Endovascular Aortic Repair for Descending Thoracic Aortic Aneurysms. Journal of Vascular Surgery, 73, 55S-83S. https://doi.org/10.1016/j.jvs.2020.05.076

[22] Hansen, N.J. (2016) Computed Tomographic Angiography of the Abdominal Aorta. Radiologic Clinics of North America, 54, 35-54.

https://doi.org/10.1016/j.rcl.2015.08.005

[23] Gudbjartsson, T., Ahlsson, A., Geirsson, A., Gunn, J., Hjortdal, V., Jeppsson, A., et al. (2020) Acute Type A Aortic Dissection-A Review. Scandinavian Cardiovascular Journal, 54, 1-13. https://doi.org/10.1080/14017431.2019.1660401

[24] Expert Panels on Vascular Imaging and Interventional Radiology, Bonci, G., Steigner, M.L., Hanley, M., Braun, A.R., Desjardins, B., Gaba, R.C., et al. (2017) ACR Appropriateness Criteria Thoracic Aorta Interventional Planning and Fol- 
low-Up. Journal of the American College of Radiology, 14, S570-S583. https://doi.org/10.1016/j.jacr.2017.08.042

[25] Flors, L., Leiva-Salinas, C., Norton, P.T., Patrie, J.T. and Hagspiel, K.D. (2013) Endoleak Detection after Endovascular Repair of Thoracic Aortic Aneurysm Using Dual-Source Dual-Energy CT: Suitable Scanning Protocols and Potential Radiation Dose Reduction. AJR. American Journal of Roentgenology, 200, 451-460. https://doi.org/10.2214/AJR.11.8033

[26] Duran, E.S., Ahmad, F., Elshikh, M., Masood, I. and Duran, C. (2019) Computed Tomography Imaging Findings of Acute Aortic Pathologies. Cureus, 11, e5534. https://doi.org/10.7759/cureus.5534

[27] Fiorucci, B., Banafsche, R., Jerkku, T., Pichlmaier, M., Kölbel, T., Rantner, B., et al. (2019) Thoracic Aortic Aneurysms-Diagnosis and Treatment Strategies. Deutsche Medizinische Wochenschrift, 144, 146-151. https://doi.org/10.1055/a-0648-0207

[28] Ridge, C.A. and Litmanovich, D.E. (2015) Acute Aortic Syndromes: Current Status. Journal of Thoracic Imaging, 30, 193-201. https://doi.org/10.1097/RTI.0000000000000155

[29] Schernthaner, R.E., Stadler, A., Beitzke, D., Homolka, P., Weber, M., Lammer, J., et al. (2012) Dose Modulated Retrospective ECG-Gated versus Non-Gated 64-Row CT Angiography of the Aorta at the Same Radiation Dose: Comparison of Motion Artifacts, Diagnostic Confidence and Signal-to-Noise-Ratios. European Journal of Radiology, 81, e585-e590. https://doi.org/10.1016/j.ejrad.2011.06.053

[30] Valente, T., Rossi, G., Lassandro, F., Rea, G., Marino, M., Muto, M., et al. (2016) MDCT Evaluation of Acute Aortic Syndrome (AAS). The British Journal of Radiology, 89, Article ID: 20150825. https://doi.org/10.1259/bjr.20150825

[31] Baliyan, V., Shaqdan, K., Hedgire, S. and Ghoshhajra, B. (2019) Vascular Computed Tomography Angiography Technique and Indications. Cardiovascular Diagnosis and Therapy, 9, S14-S27. https://doi.org/10.21037/cdt.2019.07.04

[32] Meyersohn, N.M., Szilveszter, B., Staziaki, P.V., Scholtz, J.E., Takx, R., Hoffmann, U., et al. (2017) Coronary CT Angiography in the Emergency Department Utilizing Second and Third Generation Dual Source CT. Journal of Cardiovascular Computed Tomography, 11, 249-257. https://doi.org/10.1016/j.jcct.2017.03.002

[33] Achenbach, S., Marwan, M., Schepis, T., Pflederer, T., Bruder, H., Allmendinger, T., et al. (2009) High-Pitch Spiral Acquisition: A New Scan Mode for Coronary CT Angiography. Journal of Cardiovascular Computed Tomography, 3, 117-121. https://doi.org/10.1016/j.jcct.2009.02.008

[34] Feuchtner, G., Goetti, R., Plass, A., Baumueller, S., Stolzmann, P., Scheffel, H., et al. (2010) Dual-Step Prospective ECG-Triggered 128-Slice Dual-Source CT for Evaluation of Coronary Arteries and Cardiac Function without Heart Rate Control: A Technical Note. European Radiology, 20, 2092-2099.

https://doi.org/10.1007/s00330-010-1794-7

[35] Geyer, L.L., Glenn, G.R., De Cecco, C.N., Van Horn, M., Canstein, C., Silverman, J.R., et al. (2015) CT Evaluation of Small-Diameter Coronary Artery Stents: Effect of an Integrated Circuit Detector with Iterative Reconstruction. Radiology, 276, 706-714. https://doi.org/10.1148/radiol.15140427

[36] Mangold, S., De Cecco, C.N., Wichmann, J.L., Canstein, C., Varga-Szemes, A., Caruso, D., et al. (2016) Effect of Automated Tube Voltage Selection, Integrated Circuit Detector and Advanced Iterative Reconstruction on Radiation Dose and Image Quality of 3rd Generation Dual-Source Aortic CT Angiography: An Intra-Individual Comparison. European Journal of Radiology, 85, 972-978. 
https://doi.org/10.1016/j.ejrad.2016.02.021

[37] Mangold, S., Wichmann, J.L., Schoepf, U.J., Poole, Z.B., Canstein, C., Varga-Szemes, A., et al. (2016) Automated Tube Voltage Selection for Radiation Dose and Contrast Medium Reduction at Coronary CT Angiography Using 3(rd) Generation Dual-Source CT. European Radiology, 26, 3608-3616.

https://doi.org/10.1007/s00330-015-4191-4

[38] Meinel, F.G., Nikolaou, K., Weidenhagen, R., Hellbach, K., Helck, A., Bamberg, F., et al. (2012) Time-Resolved CT Angiography in Aortic Dissection. European Journal of Radiology, 81, 3254-3261. https://doi.org/10.1016/j.ejrad.2012.03.006

[39] Sommer, W.H., Becker, C.R., Haack, M., Rubin, G.D., Weidenhagen, R., Schwarz, F., et al. (2012) Time-Resolved CT Angiography for the Detection and Classification of Endoleaks. Radiology, 263, 917-926. https://doi.org/10.1148/radiol.12111217

[40] Budde, R.P., Kluin, J., van Herwerden, L.A. and Prokop, M. (2011) Comprehensive Dynamic Aortic and Cardiac Analysis by 256-Slice Computed Tomography in Type A Dissection. The Annals of Thoracic Surgery, 92, e47-e48. https://doi.org/10.1016/j.athoracsur.2011.03.126

[41] Maldjian, P.D. and Partyka, L. (2012) Intimal Tears in Thoracic Aortic Dissection: Appearance on MDCT with Virtual Angioscopy. AJR. American Journal of Roentgenology, 198, 955-961. https://doi.org/10.2214/AJR.11.7327

[42] Singh, N., Goel, P. and Singh, Y. (2018) Pictorial Essay: Computed Tomography Findings in Acute Aortic Syndromes. SA Journal of Radiology, 22, 1309. https://doi.org/10.4102/sajr.v22i1.1309

[43] Han, J., Xiang, H., Ridley, W.E. and Ridley, L.J. (2018) Aortic Beak Sign: Aortic Dissection. Journal of Medical Imaging and Radiation Oncology, 62, 14. https://doi.org/10.1111/1754-9485.03 12785

[44] Han, J., Xiang, H., Ridley, W.E. and Ridley, L.J. (2018) Aortic Webs and Cobwebs: Aortic Dissection and Arteriopathies. Journal of Medical Imaging and Radiation Oncology, 62, 15-16. https://doi.org/10.1111/1754-9485.04_12785

[45] Liu, D., Liu, J., Wen, Z., Li, Y., Sun, Z., Xu, Q., et al. (2017) 320-Row CT Renal Perfusion Imaging in Patients with Aortic Dissection: A Preliminary Study. PLoS ONE, 12, e0171235. https://doi.org/10.1371/journal.pone.0171235

[46] Kapoor, V., Ferris, J.V. and Fuhrman, C.R. (2004) Intimomedial Rupture: A New CT Finding to Distinguish True from False Lumen in Aortic Dissection. AJR. American Journal of Roentgenology, 183, 109-112. https://doi.org/10.2214/ajr.183.1.1830109

[47] Strobl, F.F., Sommer, W.H., Haack, M., Nikolaou, K., Meimarakis, G., Koeppel, T.A., et al. (2013) Computed Tomography Angiography as the Basis for Optimized Therapy Planning before Endovascular Aneurysm Repair (EVAR). Der Radiologe, 53, 495-502. https://doi.org/10.1007/s00117-012-2450-9

[48] Sun, Z. (2011) Multislice Computed Tomography Angiography in the Diagnosis of Cardiovascular Disease: 3D Visualizations. Frontiers of Medicine, 5, 254-270. https://doi.org/10.1007/s11684-011-0153-7

[49] Hiratzka, L.F., Bakris, G.L., Beckman, J.A., Bersin, R.M., Carr, V.F., Casey, D.E., et al. (2010) ACCF/AHA/AATS/ACR/ASA/SCA/SCAI/SIR/STS/SVM Guidelines for the Diagnosis and Management of Patients with Thoracic Aortic Disease. A Report of the American College of Cardiology Foundation/American Heart Association Task Force on Practice Guidelines, American Association for Thoracic Surgery, American College of Radiology,American Stroke Association, Society of Cardiovascular Anesthesiologists, Society for Cardiovascular Angiography and Interven- 
tions, Society of Interventional Radiology, Society of Thoracic Surgeons, and Society for Vascular Medicine. Journal of the American College of Cardiology, 55, e27-e129. https://doi.org/10.1016/j.jacc.2010.02.015

[50] Paulraj, S., Ashok Kumar, P., Uprety, A. and Chaudhuri, D. (2020) Aortic Dissection and Multimodality Imaging. Echocardiography, 37, 1485-1487.

https://doi.org/10.1111/echo.14820

[51] Bossone, E., Czerny, M., Lerakis, S., Rodríguez-Palomares, J., Kukar, N., Ranieri, B., et al. (2021) Imaging and Biomarkers in Acute Aortic Syndromes: Diagnostic and Prognostic Implications. Current Problems in Cardiology, 46, Article ID: 100654. https://doi.org/10.1016/j.cpcardiol.2020.100654

[52] Chaddha, A., Eagle, K.A., Patel, H.J., Deeb, G.M., Yang, B., Harris, K.M., et al. (2019) The Clinical Impact of Imaging Surveillance and Clinic Visit Frequency after Acute Aortic Dissection. Aorta, 7, 75-83. https://doi.org/10.1055/s-0039-1692187

[53] Ziza, V., Canaud, L., Molinari, N., Branchereau, P., Marty-Ané, C. and Alric, P. (2016) Thoracic Endovascular Aortic Repair: A Single Center's 15-Year Experience. The Journal of Thoracic and Cardiovascular Surgery, 151, 1595-1603.e7. https://doi.org/10.1016/j.jtcvs.2015.12.030

[54] Iribarne, A., Keenan, J., Benrashid, E., Wang, H., Meza, J.M., Ganapathi, A., et al. (2017) Imaging Surveillance after Proximal Aortic Operations: Is it Necessary? The Annals of Thoracic Surgery, 103, 734-741. https://doi.org/10.1016/j.athoracsur.2016.06.085

[55] Zhou, Y., Wang, W.C., Zhang, X.M., Yang, C., Zheng, J., Yang, L., et al. (2018) Aortic Remodelling after Thoracic Endovascular Aortic Repair for Acute and Subacute Type B Aortic Dissection. Quantitative Imaging in Medicine and Surgery, 8, 391-398. https://doi.org/10.21037/qims.2018.05.03

[56] Ghazy, T., Mahlmann, A., Fajfrova, Z., Darwish, A., Eraqi, M., Hegelmann, H., et al. (2017) Anastomotic Leak after Surgical Repair of Type A Aortic Dissection-Prevalence and Consequences in Midterm Follow-Up. VASA, 46, 377-382. https://doi.org/10.1024/0301-1526/a000644

[57] Kaneyuki, D., Mogi, K., Watanabe, H., Otsu, M., Sakurai, M. and Takahara, Y. (2020) The Frozen Elephant Trunk Technique for Acute Retrograde Type A Aortic Dissection: Preliminary Results. Interactive Cardiovascular and Thoracic Surgery, 31, 813-819. https://doi.org/10.1093/icvts/ivaa199

[58] Vardhanabhuti, V., Nicol, E., Morgan-Hughes, G., Roobottom, C.A., Roditi, G., Hamilton, M.C., et al. (2016) Recommendations for Accurate CT Diagnosis of Suspected Acute Aortic Syndrome (AAS) - On Behalf of the British Society of Cardiovascular Imaging (BSCI)/British Society of Cardiovascular CT (BSCCT). The British Journal of Radiology, 89, Article ID: 20150705.

https://doi.org/10.1259/bjr.20150705

[59] Salmasi, M.Y., Hartley, P., Hussein, M., Jarral, O., Pepper, J., Nienaber, C., et al. (2020) Diagnosis and Management of Acute Type-A Aortic Dissection in Emergency Departments: Results of a UK National Survey. International Journal of Cardiology, 300, 50-59. https://doi.org/10.1016/j.ijcard.2019.09.074 